\title{
FICHTE/LEVINAS. UN RENDEZ-VOUS MANQUÉ AUTOUR DE L'ALTÉRITÉ
}

\author{
FICHTE/LEVINAS. A MISSED ENCOUNTER AROUND \\ THE OTHERNESS
}

\section{FICHTE/LEVINAS. UN ENCUENTRO FALLIDO EN RELACIÓN A LA ALTERIDAD}

\section{CRISTÓBAL BALBONTIN-GALLO*}

RÉsumÉ: On le sait bien, Levinas propose une pensée de l'autre, qui comme absolu ou infini invite à la subjectivité à une relation sans commune mesure qui est constitutive de la liberté du sujet. Or on peut aussi rencontrer chez Fichte cette figure de la priorité de l'Autre dans sa Doctrine de la Science en 1794 où - à propos de la théorie de la reconnaissance intersubjective - il développe cette priorité de l'altérité d'autrui dans le processus de constitution sociale de la subjectivité. Ainsi le propos de cet article est de revenir sur les deux penseurs pour esquisser les points de rapprochements ainsi que les différences qui mesurent la distance aussi bien que les fractures entre Levinas et Fichte.

MoTs CLÉs: Intersubjectivité, altérité, reconnaissance, éthique, Fichte, Levinas.

Aвstract: As it is known, Levinas proposes a thought of the otherness that, as absolute or infinite, invites the subjectivity to a relationship without common measure, constitutive of the freedom of the subjectivity. Now, not only in Levinas but also in Fichte it is possible to find this figure of otherness. In fact, he develops it concretely in his 1794 Doctrine of Science, regarding his theory of recognition. The purpose of this article then is to return to these two thinkers to outline both the points of approach and the differences that measure the distance and the fracture between them.

KEYwORDs: Intersubjectivity, alterity, recognition, ethics, Fichte, Levinas.

Resumen: Como es sabido, Levinas propone un pensamiento de la alteridad que, en cuanto absoluto o infinito, invita a la subjetividad a una relación sin medida común, constitutiva de su libertad. Ahora bien, no solo en Levinas sino también en Fichte es posible encontrar esta figura de la alteridad. En efecto, él la desarrolla concretamente en su Doctrina de la Ciencia de 1794, a propósito de su teoría del reconocimiento intersubjetivo. El propósito de este artículo entonces es volver a estos dos pensadores

\footnotetext{
* Doctor en Filosofía. Investigador asociado de IREPH Université Paris-Nanterre y académico de la Universidad Austral, Valdivia, Chile. Correo electrónico: cbalbonting@gmail.com. Orcid: https://orcid. org/0000-0001-6604-2957
} 
para bosquejar tanto los puntos de acercamiento como las diferencias que miden la distancia y la fractura entre Levinas y Fichte.

Palabras Clave: Intersubjetividad, alteridad, reconocimiento, ética, Fichte, Levinas.

Recibido: 31.01.2020. Aceptado: 7.05.2021.

\section{INTRODUCTION}

Ans La tRACE De L'AUTRE paru dans la revue Tijdschrift voor Filosofie et repris dans le recueil En découvrant lexistence avec Husserl et Heidegger, Emmanuel Levinas (2001) écrit:

La philosophie est atteinte, depuis son enfance, d'une horreur de l'Autre qui demeure Autre, d'une insurmontable allergie. C'est pour cela qu'elle est essentiellement une philosophie de lêtre, que la compréhension de lêtre est son dernier mot et la structure fondamentale de l'homme. C'est pour cela aussi quelle devient la philosophie de l'immanence et de l'autonomie ou, athéisme. Le Dieu des philosophes, d'Aristote à Leibniz, à travers le Dieu des scolastiques- est un dieu adéquat à la raison, un dieu compris qui ne saurait troubler l'autonomie de la conscience, se retrouvant elle-même à travers toutes ses aventures, retournant chez soi comme Ulysse qui, à travers toutes ses pérégrinations, ne va que vers son île natale. La philosophie qui nous est transmise attribue à ce retour non seulement la pensée théorétique, mais tout mouvement spontané de la conscience. Non seulement le monde compris par la raison cesse dêtre autre car la conscience s'y retrouve, mais tout ce qui est attitude de la conscience, c'est-à-dire valorisation, sentiment, action, travail et, d'une façon plus générale engagement, est en dernière analyse conscience de soi, c'est-à-dire identité et autonomie. La philosophie de Hegel représente l'aboutissement logique de cette allergie foncière de la philosophie. (p. 263)

Ainsi, pour Levinas, l'ensemble de la pensée métaphysique qui sétend de Platon à Hegel s'emploierait à réduire au «Même» tout ce qui s’oppose à elle comme «Autre». Autrement dit, l'histoire de la métaphysique ne serait que la marche vers une autonomie totalitaire, vers un stade où rien ne viendrait limiter la pensée et où, par conséquent, la pensée serait libre. Face à cela, Levinas propose une pensée de l'autre comme absolu ou infini où l'irruption du visage d'autrui m'invite à une relation avec une altérité irréductible, «sans commune mesure avec un pouvoir qui s'exerce» (Levinas, 1961, p. 172).

On peut en être surpris car le besoin de préserver l'altérité de l'autre était 
très en vogue dans l'idéalisme allemand. Déjà Jacobi (1987), dans une de ses lettres à Fichte, signale que l'idéalisme transcendantal (au moins dans la formulation que lui avait donné Kant) était hanté par le risque de réduire «l'Autre» au «Même», par le biais d'une pensée où la conscience ne connaît que les produits de sa propre activité. À son tour, Fichte revendiquera l'importance de «l'Autre» dans sa philosophie théorique ainsi que dans sa philosophie pratique (Sandkaulen, 2019). En effet, dès la publication de sa Doctrine de la Science (1794), Fichte précise que la constitution du sujet transcendantal passe par la rencontre de l'autre, le Anstoss, c'est-àdire le Non-Moi à partir duquel le Moi se pose comme identité face à une différence. Ainsi, chaque détermination du «Moi», chaque développement d'une unité supérieure de la conscience sera le résultat d'une contradiction avec l'autre que la conscience narrive pas à absorber. Fichte le signale ainsi dans son Fondement du droit naturel selon les principes de la science:

La question était: comment le sujet peut-il se trouver lui-même comme un objet? (...) Pour se trouver comme objet (de sa réflexion), il ne pouvait pas se trouver comme se déterminant à la spontanéité (...), mais comme déterminé à celle-ci par un choc (Anstoss) extérieur qui doit cependant lui laisser son entière liberté de se déterminer lui-même. (1984, p. 49)

Or, cette place privilégiée de l'extériorité de «l'Autre» chez Fichte peut se relever aussi à propos de la reconnaissance dans son Fondement $d u$ droit naturel selon les principes de la Doctrine de la science publié pour la première fois en 1796-1797. Il s'agit donc de revenir à ce rendez-vous manqué entre Levinas et Fichte à propos de la théorie de la reconnaissance pour déceler les conséquences que, peut-être, Levinas n’aurait pas suffisamment considérées ${ }^{1}$.

\section{LEVINAS ET SA CRITIQUE DE LA RECONNAISSANCE INTERSUBJECTIVE}

La critique que Levinas porte à l'encontre de l'ensemble de l'histoire de la métaphysique ontologique vise à dénoncer la négation de toute différence que ce soit dans l'unité de la vie, de l'idée, du savoir ou du concept. Il en va de même pour la conscience comme point d'aboutissement de

\footnotetext{
${ }^{1}$ C’est n'est pas le problème à traiter ici qui fait l'originalité de cet article, mais plutôt l'effort pour esquisser l'actualité de la pensée de Fichte avec une nouvelle grille de lecture. Par rapport à la littérature existante: Cfr. Radrizzani, I. (2010); Trescher, S. (2019).
} 
tout phénomène. Levinas signale à ce propos: «La conscience consiste à thématiser à travers une multiplicité et à manifester ainsilêtre en proclamant l'unité et l'identité» (2001, p. 319). L'identité du réel avec le rationnel dans la pensée trouve donc sa détermination dans lidentité de la conscience avec soi: «Moi = Moi» dont est solidaire l'identité logique: $« \mathrm{~A}=\mathrm{A} »$. Il en va de même pour Fichte.

En effet, chez Fichte, le «Moi = Moi» implique l'intuition de l'activité de la conscience comme objet, c'est-à-dire que le sujet se prend comme objet de sa propre conscience, à la fois réelle et idéale. Dès lors, toute forme de connaissance serait possible à partir de cette identité originale $\mathrm{du}$ «Moi = Moi», voire à partir de cette activité productrice du «Moi» où l'absolu devient «sujet = objet» et où le Moi représente cette identité. Il s'agirait là d'un principe universel par lequel les opposés pourraient être rendus harmoniques. Voilà la grande avancée de Fichte par rapport à la philosophie kantienne; il la détaillera dans sa Doctrine de la Science (1794). Kant avait nié tout passage par voie régressive dans le domaine théorique que permettait un passage du fini à l'infini. Fichte, à sa façon, suit Kant notamment lorsqu'il signale dans sa Doctrine de la Science que le Moi ne peut pas se poser sans sopposer à un Non-Moi. Il en résulte que la nature ne peut se manifester sans être déterminée par la force négative du Moi. De même, le Moi est déterminé concrètement par la nature et réciproquement la nature par le Moi. Ainsi, l'intuition intellectuelle chez Fichte s'engage dans un jeu dopposition par lequel le Non-Moi détermine un certain contenu du Moi et par lequel le Moi s'affirme comme identité face à un Non-Moi. Ainsi, Fichte va plus loin que Kant en considérant le principe d'intuition intellectuelle comme un principe d'identité absolue qui conditionne tout savoir, voire comme un principe absolu tendant à résoudre $a$ priori toutes les oppositions sujet-objet.

Dans les très rares passages de l'œuvre de Levinas où il fait référence explicite à Fichte, il lui reproche aussi ce geste philosophique caractéristique de la pensée occidentale qui cherche à réduire «l'Autre» au «Même». Ainsi il écrit: «L'équivalence de la volonté libre et de l'infini sans transcendance inspirera la pensée de l'infini chez Fichte, Schelling et Hegel» (Levinas, 1995, p. 70). De même, il signale:

La subjectivité dès lors se montre comme Moi, capable de présent, capable de commencement, acte d'intelligence et de liberté remontant au principe et au commencement, sujet opposé à l'objet, Moi qui, pour un Fichte est origine de lui-même. Absolu pensable comme tel dans 
l'oubli du Dire, dans l'apparaître ou dans 1'ordre assemblable du thème et du Dit. (Levinas, 1974, p. 207)

Sans doute Levinas fait-il allusion à l'intuition intellectuelle chez Fichte comme principe ontologique que l'on trouve à la base de l'idéalisme allemand. Il en est ainsi dans l'idéalisme transcendantal de Kant et également chez Hegel -comme esprit- ou même chez Schelling² - comme absolu. Ces deux penseurs auraient radicalisé l'intuition intellectuelle fichtéenne dans sa portée universelle. Quoi qu'il en soit, la conscience s'avère porteuse, dans son solipsisme, d'un principe d'identité qui se trouve à la base de la vérité comme res adequatio intellectus et qui finalement est redevable de sa position de maîtrise sur le monde qui ne cherche quà «coïncider avec soi». Ce principe méconnaît, dès lors, toute extériorité (Ausschlissen alles andern aus sich). Il va de même pour la reconnaissance intersubjective de Fichte.

En effet, bien que toujours de façon implicite, Levinas critique la reconnaissance intersubjective avec des arguments qui visent surtout l'Anerkennung de Hegel tout en restant valables aussi à l'égard de Fichte. Pour ce qui est de la reconnaissance, du point de vue de Levinas, Fichte n'affirme l'identité de la conscience qu'en excluant l'autre. Face à l'irréductibilité de l'autre lévinassien: «tout ce qui est dans la conscience ne serait pas posé par la conscience» (Levinas, 1974, p. 127), «contrairement à la proposition qui paraissait fondamentale à Fichte» (p. 127), où tout finalement était posé pour la conscience. Concrètement, l'économie de la reconnaissance s'accomplit à travers l'ouverture de la conscience dans une extériorité pour la surmonter et se rencontrer dans cette extériorité qui devient égale à soi-même comme intériorité. À ce propos, Levinas écrit: «ne rien recevoir d'autrui sinon ce qui est en Moi, comme si, de toute éternité se posséderait ce qui me vient du dehors» (1961, p. 14). Il s'agit donc d'un mouvement égologique qui va de Moi à Moi et qui ne fait d’autrui qu'un passage à soi. En effet, il explique: «Son premier mouvement est négatif: il consiste à retrouver en soi et à épuiser le sens d'une extériorité» (1961, p. 98). «Chacune exige la reconnaissance par l'autre pour être elle-même» (1993, p. 93). Ainsi, on a affaire à une "Conscience de soi se reconnaissant pensée infinie 'sans autre'" (Levinas, 1995, p. 34). La conscience s'affirme donc dans un narcissisme. Désormais, cette conscience de soi dans la reconnaissance apparait comme

\footnotetext{
${ }^{2}$ Emmanuel Cattin (2000) commenta ainsi par rapport à Schelling : «Lêtre et le connaître ne sont pas co-originaires seulement, mais un seul: l'être de l'identité absolue n'est que la connaissance de soi de celle-ci» (p. 19).
} 
nécessairement orientée vers l'affirmation de soi à travers la suppression de toute altérité.

En outre, un passage important de la théorie de la reconnaissance soumis à la critique de Levinas concerne la réciprocité. Celle-ci marque, dans l'Encyclopédie hégélienne, le passage à la dimension universelle de l'esprit. Or dans le Fondement du droit naturel de Fichte elle constitue la dimension objective de la liberté qui permet d'équilibrer celle-ci dans l'extériorité aussi bien que dans l'intériorité du sujet. Pourtant, Levinas sera également critique vis-à-vis de la réciprocité à l'intérieur de la reconnaissance intersubjective. D’abord, parce que la réciprocité implique d'introduire une symétrie éthique qui risque deéliminer la différence irréductible de l'autre et de la rabaisser à une position d'alter ego qui implique la possibilité de faire place à une substitution de «Moi» à «Toi» et de «Toi» à «Moi». En effet, Levinas (1995) signale à ce propos:

Mon interrogation consistait à mettre en question cette réciprocité initiale. Autrui à qui je madresse, ne serait-il pas d'abord celui envers qui j’ai la relation que l'on a à légard de celui qui est plus faible. Par exemple : je suis généreux envers autrui sans que cette générosité soit aussitôt réclamée comme réciproque. Bien que Buber soit l'un des premiers penseurs à mettre l'accent sur une relation du Je-Tu par rapport au Jecela, ce concept de réciprocité me troublait parce que dès lors que l’on est généreux en espérant la réciprocité, cette relation ne relève plus de la générosité mais de la relation commerciale, léchange des bons procédés. (p. 111)

Ce faisant, la réciprocité fait violence à l'asymétrie d'autrui qui est la dimension permettant de préserver la portée éthique de la responsabilité. Autrement dit, seule la responsabilité à l'égard d'autrui sans caution de réciprocité permet de préserver la libéralité et empêche le désintéressement éthique de tomber dans une simple logique de calcul de gains et de pertes où «nul n'est assigné à répondre sans garantie de retour, nul ne se commet avec ce qui sera peut-être une perte sans contrepartie» (Chalier, 2006, p. 58). Cette symétrie de perspectives implicites à la réciprocité et qui ne saurait empêcher suffisamment l'altérité d’autrui de plonger dans un fond commun, cherche à surmonter toute différence dans le "Même».

Or, la détermination de la reconnaissance comme réciprocité n’est comprise que dans la perspective d'un troisième terme: l'horizon de la vérité et l'économie du savoir métaphysique qu'elle comporte. En effet, il s'agit d'une conscience qui doit se saisir elle-même dans l'objectivité comme vérité et devenir ainsi principe de toute connaissance. Dès lors, ce principe 
de tout savoir ne se réfère pas seulement à la connaissance, mais comporte également une détermination sur l'ensemble du réel. C'est donc une ontologie dans la pleine coïncidence de lêtre et de l'essence que la théorie de la reconnaissance intersubjective comporte. C'est une véritable odyssée de la liberté dans la connaissance vers un stade irréductible où rien ne viendra limiter la pensée. Ainsi la reconnaissance, qui se veut un moment d’ouverture à l'altérité, est trahie par ses présupposés ontologiques qui comprennent tout autre comme un moment de vérité. Il va ainsi de Fichte chez qui il s'agit d'assurer le passage du Moi concret vers un Moi universel et absolu sous la forme d'une intuition intellectuelle qui rend la connaissance ainsi que toute pensée de l'essence de lềtre possible.

Face à cela, il s'agit justement pour Levinas d'opposer la socialité pacifique issue de la rencontre de l'Autre à léconomie de la reconnaissance. En effet, Levinas (1991) écrivit: «La rencontre d’autrui consiste dans le fait que malgré l'étendue de ma domination sur lui et de sa soumission, je ne le possède pas» (p. 20). Il s'agit de reconsidérer la signifiance d'une certaine faiblesse humaine dans l'expérience du visage d'autrui; signifiance qui implique la revendication de l'hétéronomie de la conscience à travers une responsabilité pour autrui qui précède la liberté. Il s’agit donc de passer de l'idéalisme d'une conscience en quête de reconnaissance, à la radicalisation de l'expérience phénoménologique d'autrui afin de rendre compte d'une relation qui déborde toute conscience thématisant. Infiniment transcendant, infiniment étranger, autrui demeure l'opposition absolue par excellence où le visage s'affirme lui-même, tel un Kath auto, c'est-à-dire comme une résistance totale à toute prise. L'irruption d’autrui implique donc une liberté inaccessible qui, s'agissant d'un Autre, est avant tout une impossibilité qui met en question cette brutale spontanéité de la conscience. Le visage d'autrui ne saurait se réabsorber dans une représentation ni non plus être compris comme une altérité relative qui, comme une comparaison d'une espèce qui s'exclurait réciproquement, se place encore dans une communauté d'un genre ou dans le point de vue d'un troisième terme. Autrement dit, il ne saurait répondre à un processus qui va du genre à lespèce. Dès lors, lordre de la logique garde le silence face à cette condition exceptionnelle d'autrui. De la même manière que l'infini va au-delà de la pensée chez Descartes, autrui est hors de toute mesure par rapport au pouvoir et à la liberté que le «Moi» exerce. Il en résulte que le visage d’autrui dans sa hauteur et dans son asymétrie impose le premier commandement «tu ne commettras pas de meurtre ». Cette disproportion ou asymétrie entre autrui et moi, à lorigine de son caractère infini ou absolu, constitue précisément léthique. 
Cependant, l'impossibilité de tuer n'a pas une signification uniquement négative et formelle. En effet, la relation avec l'infini conditionne la conscience positivement. L'infini se présente, se lève dans la nudité et la misère d'un visage qui brise ses formes plastiques pour se retrouver aux confins du monde. Levinas (1994) dira: «Lépiphanie de l'absolument autre est visage où l'Autre m'interpelle et ordonne de par sa nudité, de par son dénuement. Il m’interpelle de son humilité et de sa hauteur» (p. 79). Toutefois, cette misère et cette humilité supposent une proximité, une socialité. Lépiphanie du visage fait appel à moi. Ainsi, je réponds à son expression et à sa révélation. La guerre donc, présuppose la paix, c'est-àdire la responsabilité préalable envers autrui.

Cette impossibilité de la totalisation nest pas purement négative. Elle dessine une relation nouvelle, un temps diachronique quaucune historiographie ne transforme en simultanéité totalisée et thématisée et dont l'accomplissement concret serait la relation de l'homme à l'homme, la proximité humaine, la paix entre les hommes, telle quaucune synthèse se produisant au-dessus de leurs têtes ou derrière leur dos ne saurait dominer (...) (Levinas, 1995, p. 68).

Dans cette droiture même du face-à-face, sans l'intermédiaire d’aucune image de sa nudité, la liberté ne s'inhibe alors non point comme heurtée par une résistance, mais comme arbitraire, coupable et honteuse. Ainsi, la conscience sélève à la responsabilité. Levinas (1974) fait une critique explicite à l'égard de Fichte à ce propos:

Liberté portée par la responsabilité qu’elle ne saurait endosser, élévation et inspiration sans complaisance - le pour l'autre du sujet ne saurait s'interpréter ni comme complexe de culpabilité (qui suppose une liberté initiale), ni comme bienveillance naturelle ou instinct divin, ni comme un je ne sais quel amour ou une je ne sais quelle tendance au sacrifice - tout à lopposé de la conception fichtéenne où toute souffrance due à laction du non-moi sur le moi est, au préalable, la position de cette action du non-moi par le moi. (p. 160)

Or, ce passage «d'un esprit à l'autre» ne se produit pas sans l'injonction d'autrui, responsabilité «enseignée par le maître» et qui se trouve de toute éternité dans la subjectivité comme désir de l’infini, voire comme désir de l'absolument autre qui anime l'intellectualisme. Ainsi, pour Levinas, autrui n'est autre que si son altérité est absolument irréductible. Dès lors, on comprend pourquoi Levinas (1974) écrit à l'encontre de Fichte: 
Comment le libre Moi fichtéen subirait-il la souffrance qui lui viendrait du non-moi ? La finitude de la liberté signifierait-elle la nécessité où se trouve une volonté de vouloir dans une situation donnée limitant l'arbitraire du vouloir ? Cela n'entame pas l'infini de la liberté au-delà de ce que la situation détermine. Dans la liberté finie se dégage donc un élément de liberté pure que la limitation n'affecte pas dans son vouloir. Ainsi, la notion de liberté finie pose plutôt qu'elle ne résout le problème d'une limitation de la liberté du vouloir. La responsabilité pour autrui - responsabilité illimitée que la rigoureuse comptabilité du libre et du non-libre ne mesure plus - réclame la subjectivité comme otage irremplaçable qu'elle dénude sous le Moi dans une passivité de persécution, de refoulement et d'expulsion hors l'essence, en Soi. (p. 159)

\section{L'ALTÉRITÉ DANS LA RECONNAISSANCE FICHTÉENNE}

Certes, Levinas (1995) fait tout de même une remarque positive à l'égard de Fichte qui se trouve dans le texte qui inaugure le recueil Altérité et transcendance:

Au cours de l'histoire de la philosophie occidentale, l'impossibilité de la totalisation elle-même a pu se manifester en de multiples occasions : dans le dualisme des forces et des valeurs opposées d'Anaximandre ; dans le bien et dans la notion d'un au-delà de l'Essence chez Platon et chez Plotin ; quant à lêtre lui-même, dans son équivocation qui n’admet que l'unité d'analogie et dans la transcendance du premier moteur; dans l'idée qui soutient la philosophie d'un dieu transcendant ne «faisant pas totalité» avec la créature; dans le Sollen fichtéen qui est non pas une simple impuissance de penser lêtre, mais un débordement de lêtre, irrécupérable par lêtre débordé et qui, en fin de compte, sauve celui-ci de l'illusion (...). (p. 67)

Pourtant, il apparaît que Levinas passe à côté de l'importance que l'altérité tient dans la reconnaissance intersubjective de Fichte ${ }^{3}$. Cest justement ceci qu'il s'agit d'élucider.

En effet, par rapportàla reconnaissance définiepar Fichte, lecheminement de son développement doit être cherché comme une réaction au formalisme abstrait de l'idéalisme transcendantal de Kant. Rappelons-nous que dans le texte Métaphysique des mours, la pensée kantienne de l'autonomie

${ }^{3}$ Sur la reconnaissance chez Fichte voir les études: Siep (1992); Vigo (2016). 
visait à comprendre l'homme comme une fin en soi, c'est-à-dire, comme fait d'une certaine nature qui ne peut être remplacée par aucune autre. La volonté de tout être raisonnable est conçue comme volonté instituant une législation morale. Cette volonté n'est pas seulement soumise à la loi mais de plus elle l'institue. Elle est donc autonomie de la volonté. L'homme est obligé d'agir selon sa volonté propre, une volonté qui doit être en accord avec la législation universelle. Cette approche législatrice et universelle est une règle objective sans contradiction et qui, par conséquent, est valable sans être soumise à des conditions subjectives ni à des contingences. La loi morale, faite de raison, soffre à nous comme proposition synthétique $a$ priori, elle s'impose à elle-même sans être fondée sur aucune intuition. En vertu de la moralité qui réside en nous, nous sommes législateurs de nousmêmes et en tant que législateurs nous nous construisons dans un royaume de liberté au sein duquel la raison pure peut être pratique. Ajoutons qu'elle seule peut être pratique de manière inconditionnée face à la causalité de la nature.

Toutefois, en critiquant Kant, Fichte met en garde sur une question antérieure à l'autonomie du sujet et dont celle-ci dépend. Cette question concerne la genèse et le développement de la conscience de soi face à d’autres consciences. Comme Fichte (1969) l'exprime fort bien:

Qu'est-ce qui autorise l'homme à appeler une partie déterminée du monde des corps son corps ? Comment en vient-il à considérer cette partie comme son corps en tant quappartenant à son Moi, alors qu'il est pourtant directement opposé à celui-ci ? (...) Comment l'homme en vient-il à admettre et à reconnaître des êtres raisonnables d'espèce en dehors de lui alors que pourtant de tels êtres ne sont absolument pas donnés immédiatement dans sa pure conscience de soi ? Je dois aujourd'hui établir la distinction de l'homme dans la société, et la solution de ce problème suppose la réponse à la dernière question. J'appelle société la relation des êtres raisonnables entre eux. Le concept de société n'est possible que si l'on présuppose qu'il y a effectivement en dehors de nous des êtres raisonnables, et que s'il existe des marques distinctives qui nous permettent de distinguer ceux-ci de tous les autres êtres qui ne sont pas raisonnables et par conséquent n’appartiennent pas à la communauté. Comment parvenons-nous à cette supposition? (p. 45)

En peu de lignes, Fichte vise l'une des critiques les plus puissantes contre l'idéalisme transcendantal de Kant et contre l'idéalisme tout court: la dénonciation du solipsisme comme une impossibilité de justifier l'existence 
d’autrui dans la prétention universelle de la pensée de l'ego transcendantal. Sa prémisse vise à ce que l'autonomie de la subjectivité ne puisse être justifiée sans hypostasier le noyau apodictique du sujet transcendantal hors du contexte des relations d'intersubjectivité. En effet, selon Fichte on ne peut soi-même se donner l'expérience de la liberté sans préalablement avoir accompli l'expérience d'une transcendance par rapport à soi. Pour cela, à un moment donné, il faut que l'ipséité se retrouve face à l'expérience de se considérer soi-même comme objet. Ce qui nous conduit à la question suivante: "Comment le sujet peut-il se retrouver lui-même comme objet ?» (Fichte, 1984, p. 49). La réponse de Fichte sur laquelle Hegel reviendra (tout comme Sartre dans la troisième partie de Lêtre et le néant), est que le sujet a besoin d'un autre sujet pour rendre compte de soi comme objet et, par ce moyen, aller au-delà de cette condition à travers la liberté. Autrement dit, soi-même ne peut se donner l'expérience dêtre à la fois sujet et objet sans une médiation intersubjective.

Les circonstances décrites plus haut mèneront Fichte à introduire pour la première fois en 1796 à travers son texte Fondement du droit naturel selon les principes de la doctrine de la science le sens moderne du concept d'Anerkennung ou reconnaissance intersubjective dans l'histoire de la philosophie. L’expression sera léguée à la postérité dans le titre du troisième paragraphe correspondant au deuxième théorème de la première section du texte dans les termes qui suivent: "Lêtre raisonnable fini ne peut pas s'attribuer à lui-même une causalité libre dans le monde sensible sans l'attribuer aussi à d'autres, par conséquent sans admettre aussi d'autres êtres raisonnables finis hors de lui» (Fichte, 1984, p. 46). Ainsi, en opposition avec le mythe privé de la liberté, le sujet ainsi que son autonomie passent par la médiation extérieure des autres. Cela implique non seulement que les autres représentent une condition transcendante de ma liberté, mais aussi que l'action positive d'autrui sur moi-même est déterminante comme possibilité de ma subjectivité et, par conséquent, de ma liberté. Fichte revient de manière brève sur ce point:

Cet être raisonnable ne doit nullement par l'appel (d'autrui) être déterminé, être soumis à une nécessité d'agir, comme, dans le concept de causalité, leffet l'est par la cause; au contraire il doit seulement, à la suite de cet appel, se déterminer lui-même à agir. $(1984$, p. 52)

La limitation de la liberté dans l'autre liberté est, paradoxalement, une évidence de la liberté dans la mesure où cette limitation suppose d’emblée la reconnaissance extérieure de la liberté. Ainsi, la limitation de la liberté 
implique une première synonymie de la translation catégorielle ou metábasis du verbe reconnaitre dans le substantif «reconnaissance».

Il résulte de cette approche une conséquence qui sera plus tard importante pour Hegel et que Fichte réaffirmera avec force, à savoir: "Le sujet doit donc, tout en posant cette limitation, en même temps avoir posé hors de lui quelque chose comme la raison déterminante» (1984, p. 51). La condition éthique décentrée du sujet est donc simultanée à une condition ontologique décentrée de cette subjectivité. Cela est bien accrédité dans ses lettres réunies dans le Tatsachen des Bewußtseins (1971), publié pour la première fois en 1810, où Fichte confirmera son jugement considérant que l'altérité d’autrui contrarie tous les présupposés de la philosophie transcendantale. Cela implique à son tour la question de la distribution du fondement de la liberté entre intériorité et extériorité du sujet. Autrement dit, comment équilibrer cette équation d'extériorité et d'intériorité de la liberté?

À ce propos Fichte plaidera non pour une solution d'opposition mais pour une solution de réciprocité et de symétrie entre le sujet et l'extériorité de la liberté. De cette façon, pour Fichte,

La relation dêtres libres les uns avec les autres est par conséquent une relation d'action réciproque par l'intelligence et la liberté. Aucun ne peut reconnaitre l'autre s'ils ne se reconnaissent pas tous les deux réciproquement; et aucun ne peut traiter l'autre comme un être libre si tous les deux ne se traitent pas ainsi réciproquement. (1984, p. 59)

La réponse du sujet face à la demande de reconnaissance de la liberté d'autrui implique donc une économie de reconnaissance réciproque qui fonde une perspective normative de responsabilité mutuelle. Léquilibre de la liberté partagée est proportionnel à cette économie de la responsabilité réciproque. Si un individu devient sujet de la liberté seulement à travers la reconnaissance d'un autre, cela implique, dans toute sa dimension générale, ce que Fichte avait bien résumé dans la phrase suivante: "L'homme ne devient homme que parmi les hommes (...)» (1984, p. 54). Autrement dit, il s'agit d’assurer un accès de la conscience de soi et à sa liberté dans l'extériorité. C'est justement à cet enjeu que veut répondre la dynamique de la reconnaissance intersubjective de Fichte où les actions réciproques des individus s'appellent mutuellement à un agir libre qui limite simultanément sa propre activité au bénéfice de l'autre. Ceci permet aux individus de développer une conscience commune d'une validité objective de leur 
subjectivité. Désormais, se sachant reconnu par un autre, le sujet découvre des aspects de sa propre identité subjective comme sujet libre. Comme Fichte l'exprime bien: «Je mauto-limite dans mon appropriation de la liberté, du fait que je laisse aussi subsister de la liberté pour d’autres. Le concept de droit est donc le concept de la relation nécessaire dêtres libres les uns avec les autres» (1984, p. 24).

Or, le point intéressant est que loin de privilégier la priorité égocentrique, Fichte a pour objectif de privilégier la reconnaissance d'autrui. Concrètement, il pose l'origine de léconomie de la reconnaissance réciproque dans l'injonction ou la demande (Aufforderung) de reconnaissance qu'autrui porte sur moi. Or, cette demande de reconnaissance, afin dêtre satisfaite, implique l'appel à la liberté du sujet pour mettre en avant cet acte de reconnaissance. Autrement dit, la demande de reconnaissance d'autrui ne peut être remplie que par l'exercice de la liberté du sujet. Comme Fichte (1984) le souligne:

Si lềtre hors de lui n’avait pas agi causalement et n'avait pas ainsi appelé le sujet à l'action causale, celui-ci n’aurait pas non plus, de lui-même, agi. (...) Elle est aussi conditionnée quant à la matière: au sujet est indiquée la sphère de son action en général. (p. 57)

Ainsi la demande de reconnaissance que porte autrui est un appel à la condition du sujet quaucun autre étant ne peut remplir.

Lappel est la matière de l'action causale, et une causalité libre de lêtre raisonnable, à quoi il invite, est son but final. (...) La finalité de cet appel est conditionnée par lentendement et par la liberté de lêtre auquel il sadresse. Cette cause doit par conséquent nécessairement avoir le concept de raison et liberté; donc elle doit elle-même être un être capable de concepts, une intelligence, et, puisque, selon ce qui a été démontré, ce n'est pas possible sans liberté, être aussi un être libre, donc en général un être raisonnable et être posée comme telle. (Fichte, 1984, p. 52)

Ainsi au fur et à mesure que jassume ma liberté sous l'injonction d'autrui, je peux répondre à sa demande de reconnaissance. De même, c'est seulement en ayant le statut de sujet que je suis en condition de répondre à l'expectative d'autrui d’être considéré comme un tel sujet. Ce faisant, la demande de reconnaissance quautrui porte sur un autre présuppose chez cet autre une certaine capacité à y répondre. Autrement dit, le sujet doit comprendre l'injonction qui lui est adressée et être capable d'y réagir. Il 
s'ensuit que la reconnaissance suppose une communauté d’acteurs rationnels mis en relation. Comme Fichte le signale à plusieurs reprises: «La cause de l'appel posée en dehors du sujet doit donc au moins supposer la possibilité que ce dernier puisse comprendre et concevoir; autrement son appel n'a absolument aucune fin» (1984, p. 52). D’ailleurs, comme il l'affirme dans son System der Sittenlehre, le sujet ne peut comprendre cette convocation à l'activité de l'autre sans l'attribuer à un être réel en dehors de lui qui veut communiquer le concept de l'action convoquée, et par conséquent, qui est capable d'un concept, c'est-à-dire un être qui soit rationnel.

Le geste de Fichte implique ainsi toute une perspective hétérocentrique de la liberté. C'est par l'appel exceptionnel d'autrui que j'accède à la condition humaine de ma liberté. Ainsi, Fichte (1984) écrit: «Mais l’objet n’est pas conçu autrement, et ne peut être conçu autrement, que comme un simple appel à agir adressé au sujet. Par conséquent, dans l'exacte mesure où le sujet conçoit cet objet, il a le concept de sa propre liberté (...)» (p. 52). Ce faisant, la demande de reconnaissance implique que ma liberté est conditionnée par la liberté d'autrui qui, à son tour, la rend possible. Dès lors, une liberté infinie constituerait une contradiction: en niant la liberté de l'autre à partir de laquelle j’ai accès à ma propre liberté, cette dernière serait également niée. Autrement dit, l'Aufforderung ou demande de reconnaissance par autrui, nest pas la cause de la liberté chez le sujet mais la source des conditions pour avancer vers l'autoconscience de cette liberté à partir de la réponse requise par l'autre. En ce sens, le concept d'Aufforderung unirait la dimension interne de la liberté du sujet avec la dimension externe donnée par l'altérité tout en préservant la contingence propre de cette altérité vis-àvis du sujet ${ }^{4}$. Bref, il s'agirait de la reconnaissance de cette liberté à travers autrui. En effet, parce qu'autrui demande ma reconnaissance, je deviens, je dois devenir, conscient de ma liberté et responsable à l'égard de cet autrui. Lautre convoque le réveil de ma conscience et exige mon activité. Ce faisant, encore une fois, Fichte opère une inversion de la priorité du sujet transcendantal au bénéfice de l'altérité. La ressemblance avec Levinas est évidente au sens d'une autonomie décentrée. Pourtant, à la différence de ce dernier, Fichte (1984) écrit: «Lêtre raisonnable fini ne peut admettre d'autres êtres raisonnables finis hors de soi, sans se poser comme se tenant avec eux dans une relation déterminée que l'on nomme la relation juridique» (p.

\footnotetext{
${ }^{4}$ Selon Nicolao Merker, Fichte souligne la portée contingente de l'autre vis-à-vis la prétention de son objectivité comme étant fondée soit dans le concept, soit comme un produit de la conscience (Merker, 1961, pp. 467-468).
} 
56). Par contre cette relation éthique chez Levinas c'est une relation sans relation, qui mesure l'idée même d'une responsabilité infinie à légard de l'autre.

Ainsi, ce que Fichte vise à démontrer c'est que laccès à la condition de sujet de droit comme agent rationnel passe à travers la reconnaissance intersubjective par laquelle les sujets se reconnaissent de façon réciproque en tant qu'agents rationnels. De cette façon, Fichte donne une assise concrète, voire phénoménologique, au Droit. En effet, le Droit objectif attribue une «personnalité» à un agent rationnel sans préciser comment se détermine ce caractère rationnel. De cette manière, la reconnaissance implique la mise en place d'un statut ontologique de l'homme, diffèrent des autres étants, qui permet de façon liminaire de trancher la question de la rationalité d'un sujet de droit. Comme Fichte (1984) le résume bien: «Corollaires, L'homme (...) ne devient homme que parmi les hommes et puisqu'il ne peut être rien d'autre qu'un homme, et qu'il n'existerait aucunement s'il nétait tel, si en général il doit y avoir des hommes, il faut qu'ils soient plusieurs» (p. 54). La rationalité des sujets n'est pas tant le résultat du concept de rationalité lui-même mais plutôt de l'expérience concrète de la reconnaissance du sujet comme protagoniste de ses actions. «La condition était que je reconnaisse lautre comme être raisonnable (d'une façon valant pour lui et pour moi), c'est-à-dire que je le traite comme tel car seule l'action est une telle reconnaissance valant universellement» $(1984$, p. 62).

\section{CONCLUSION}

Tout en étant d'accord avec Levinas pour dénoncer l'économie violente qui suppose la reconnaissance de soi par l'autre, ne peut-on pas considérer qu'il a passé sous silence la portée éthique de la reconnaissance de l'autre par soi chère à Fichte ? En effet, pourquoi nier la possibilité à autrui de se rapprocher, de venir à la lumière, à la visibilité, et par là, de réclamer une identité et une dignité parmi les hommes? Pourquoi nier la portée éthique de la gratuité d'un acte de reconnaissance offert sans contrepartie ? Autrement dit, pourquoi nier cette affirmation d'autrui par et pour soi, Kath auto, par laquelle il demande à se faire reconnaitre? Notons qu'autrui ne peut prendre la parole non pas à travers un langage privé mais seulement à travers un langage public qui est l'expression d'un ordre social déjà constitué et qui lui permet justement de se mettre en rapport avec les autres. Ainsi, la pensée qui confine autrui dans son invisibilité, dans la non-lumière, nest pas vraiment 
respectueuse de l'altérité. En effet, elle empêche la possibilité d’apparaître dans une dimension publique, dêtre susceptible d'une personnalité et, de ce fait, dêtre sujet de droits et de devoirs en société, ce que Fichte à bien saisi dans la portée normative de la reconnaissance intersubjective. Derrida a donc tout à fait raison de remarquer dans Violence et métaphysique qu'autrui se trouve à la fois plus loin mais aussi plus proche que n'importe quelle autre composante de notre vie. Ainsi, il apparait qu'il y a une portée éthique dans la demande de reconnaissance d'identité que l'autre affirme de soi et dont il tire un sentiment de valeur et de dignité parmi les hommes. L'identité personnelle implique également de laisser place à une différence. Il en est ainsi parce que l'autre peut prendre la parole pour se rapprocher de nous et passer du «cri du besoin à l'expression du désir» (Derrida, 1967, p. 219). C'est une visibilité sans laquelle la condition humaine se verrait diminuée. Par ailleurs, la possibilité de la reconnaissance d’autrui n'implique pas de nier la différence mais justement de la consacrer en l'opposant à un ineffable mystère. C'est justement ce que Levinas refuse catégoriquement en plaçant un A majuscule pour souligner la transcendance infinie de l'Autre et empêcher, in extremis, toute possibilité de le penser dans une totalité. Lautre lévinassien est ainsi une pure ouverture qui risque de placer l'éthique de l'altérité aux limites des possibilités de toute éthique.

En synthèse, nous ne pouvons que confirmer l'affirmation de Derrida (1967) lorsqu'il signale que la responsabilité à l'égard d'autrui «ne peut se dire que dans le langage du Même» (p. 165). Or, il y a une portée éthique dans la dissymétrie dans laquelle la différence d’autrui est aussi véritablement respectée. Dire «dissymétrie» ne revient pas à dire «asymétrie». En effet, la dissymétrie ne va pas sans une certaine symétrie tout comme la «démesure» ne va pas sans une certaine «mesure». Pourtant, Levinas qualifie l'altérité de l'Autre comme «incommensurable» et comme «asymétrique» et de ce fait l'altérité dissymétrique d’autrui se voit elle-même violentée. Or, préserver le «décentrement éthique de la subjectivité par l'autre» ainsi que la «dissymétrie» d'autrui est, d'une certaine manière, tout l'enjeu de la reconnaissance de l'autre.

\section{RÉFÉRENCES}

Cattin, E. (2000). Le Tournant. Indications sur le Darstellung de 1801. En F.W.J. Schelling. Exposition de mon système de la philosophie (pp. 7-26). Paris: Vrin. Chalier, C. (2006). Figures du féminin ( $2^{a}$ edic.). Paris: Des femmes. 
Derrida, J. (1967). Violence et métaphysique. Lécriture et la différence. Paris: Seuil.

Fichte, J.G. (1794). Über den Begriff der Wissenschaftslehre oder der sogenannten Philosophie. Weimar: Industrie-Comptoir.

Fichte, J.G. (1969). La destination du savant, traduit par J.L. Vieillard-Baron. Paris: Vrin.

Fichte, J.G. (1971). Tatsachen des Bewußtseins. Fichtes Werke, Band 2. Berlin: Walter de Gruyter Verlag.

Fichte, J.G. (1984). Fondement du droit naturel selon les principes de la doctrine de la science. Paris: PUF.

Fichte, J.G. (1995). Das System der Sittenlehre nach den Prinzipien der Wissenschaftslehre. Hamburg: Meiner.

Hegel, G.W.F. (1988). L'Encyclopédie des sciences philosophiques. Troisième partie: Philosophie de l'esprit (Trad. de Bernard Bourgeois). Paris: Vrin.

Jacobi (1987). Brief an Fichte (1799). En E. Behler (ed.). Philosophy of German Idealism (pp. 119-141). New York: Continuum.

Kant, I. (1993). Fondements de la métaphysique de mours (Trad. de Victor Delbos). Paris: LGF.

Levinas, E. (1961). Totalité et infini. La Haye: Martinus Nijhoff.

Levinas, E. (1974). Autrement quêtre ou au-delà de l'essence. La Haye: Martinus Nijhoff.

Levinas, E. (1991). Entre nous. Essais sur le penser-à-l’autre. Paris: Grasset.

Levinas, E. (1993). Dieu, la mort et le temps. Paris: Grasset.

Levinas, E. (1994). Liberté et commandement. Paris: Fata Morgana.

Levinas, E. (1995). Altérité et transcendance. Paris: Fata Morgana.

Levinas, E. (2001). En découvrant l'existence avec Husserl et Heidegger (3a edic.). Paris: Vrin.

Merker, N. (1961). Le origini della logica hegeliana. Milano: Feltrinelli.

Radrizzani, I. (2010). Le Même, l'Autre et la Limite chez Fichte et chez Levinas. Archives de Philosophie 73(2), 285-295.

Sandkaulen, B. (2019). Ichheit und Person. Zur Aporie der Wissenschaftlehre in der Debatte zwischen Fichte und Jacobi. En Über den Widerspruch zwischen System und Freiheit. Jacobis Philosophie (pp. 201-224). Hamburg: Meiner Verlag.

Siep, L. (1992). Praktische Philosophie in Deustche Idealismus. Frankfurt am Main: Suhrkamp Verlag.

Trescher, S. (2019). Leiblichkeit und Gottesbeziehung: Eine Strukturanalyse ausgehend von Fichte und Levinas. Freiburg: Karl Alber Verlag.

Vigo, A. (2016). Identidad práctica y reconocimiento. El debate contemporáneo y el modelo fichteano. Metafísica y persona, 15, 11-46. 